\title{
Fat-induced membrane cholesterol accrual provokes cortical filamentous actin destabilisation and glucose transport dysfunction in skeletal muscle
}

\author{
K. M. Habegger • B. A. Penque • W. Sealls • L. Tackett • L. N. Bell • E. K. Blue • \\ P. J. Gallagher • M. Sturek • M. A. Alloosh • H. O. Steinberg • R. V. Considine • \\ J. S. Elmendorf
}

Received: 13 July 2009 /Accepted: 19 September 2011 /Published online: 15 October 2011

(C) The Author(s) 2011. This article is published with open access at Springerlink.com

\begin{abstract}
Aims/hypothesis Diminished cortical filamentous actin (F-actin) has been implicated in skeletal muscle insulin resistance, yet the mechanism(s) is unknown. Here we tested the hypothesis that changes in membrane cholesterol could be a causative factor, as organised F-actin structure emanates from cholesterol-enriched raft microdomains at the plasma membrane.

Methods Skeletal muscle samples from high-fat-fed animals and insulin-sensitive and insulin-resistant human participants were evaluated. The study also used L6 myotubes to directly determine the impact of fatty acids (FAs) on membrane/ cytoskeletal variables and insulin action.
\end{abstract}

Electronic supplementary material The online version of this article (doi:10.1007/s00125-011-2334-y) contains peer-reviewed but unedited supplementary material, which is available to authorised users.

K. M. Habegger $\cdot$ B. A. Penque $\cdot$ W. Sealls $\cdot$ L. Tackett $\cdot$

L. N. Bell $\cdot$ E. K. Blue $\cdot$ P. J. Gallagher $\cdot$ M. Sturek •

M. A. Alloosh $\cdot$ R. V. Considine $\cdot$ J. S. Elmendorf $(\square)$

Department of Cellular and Integrative Physiology,

Indiana University School of Medicine,

VanNuys Medical Science Building Rm 308A,

635 Barnhill Drive,

Indianapolis, IN 46202-5120, USA

e-mail: jelmendo@iupui.edu

K. M. Habegger · J. S. Elmendorf

Department of Biochemistry and Molecular Biology,

Indiana University School of Medicine,

Indianapolis, IN, USA

K. M. Habegger • B. A. Penque - W. Sealls $\cdot$ L. N. Bell

E. K. Blue • P. J. Gallagher • M. Sturek • M. A. Alloosh •

R. V. Considine $\cdot$ J. S. Elmendorf

Indiana University School of Medicine Center

for Diabetes Research,

Indianapolis, IN, USA
Results High-fat-fed insulin-resistant animals displayed elevated levels of membrane cholesterol and reduced F-actin structure compared with normal chow-fed animals. Moreover, human muscle biopsies revealed an inverse correlation between membrane cholesterol and wholebody glucose disposal. Palmitate-induced insulin-resistant myotubes displayed membrane cholesterol accrual and F-actin loss. Cholesterol lowering protected against the palmitate-induced defects, whereas characteristically measured defects in insulin signalling were not corrected. Conversely, cholesterol loading of L6 myotube membranes provoked a palmitate-like cytoskeletal/GLUT4 derangement. Mechanistically, we observed a palmitate-induced

K. M. Habegger $\cdot$ B. A. Penque $\cdot$ W. Sealls $\cdot$ J. S. Elmendorf Indiana University School of Medicine Center for Membrane Biosciences, Indianapolis, IN, USA

W. Sealls $\cdot$ M. Sturek $\cdot$ M. A. Alloosh · J. S. Elmendorf Indiana University School of Medicine Center for Vascular Biology and Medicine,

Indianapolis, IN, USA

L. N. Bell $\cdot$ R. V. Considine

Department of Medicine, Division of Endocrinology and Metabolism, Indiana University School of Medicine, Indianapolis, IN, USA

H. O. Steinberg

Merck Research Laboratories,

Rahway, NJ, USA 
increase in O-linked glycosylation, an end-product of the hexosamine biosynthesis pathway (HBP). Consistent with HBP activity affecting the transcription of various genes, we observed an increase in Hmgcr, a gene that encodes 3-hydroxy-3-methyl-glutaryl coenzyme A reductase, the rate-limiting enzyme in cholesterol synthesis. In line with increased HBP activity transcriptionally provoking a membrane cholesterol-based insulin-resistant state, HBP inhibition attenuated $\mathrm{Hmgcr}$ expression and prevented membrane cholesterol accrual, F-actin loss and GLUT4/glucose transport dysfunction.

Conclusions/interpretation Our results suggest a novel cholesterolgenic-based mechanism of FA-induced membrane/cytoskeletal disorder and insulin resistance.

Keywords Actin · Cholesterol · Fatty acid · GLUT4 · Insulin resistance $\cdot$ Membrane $\cdot$ Palmitate $\cdot$ Skeletal muscle

$\begin{array}{ll}\text { Abbreviations } & \\ \text { DON } & \text { 6-Diazo-5-oxo-L-norleucine } \\ \text { FA } & \text { Fatty acid } \\ \text { F-Actin } & \text { Cortical filamentous actin } \\ \text { GSV } & \text { GLUT4 storage vesicle } \\ \text { HBP } & \text { Hexosamine biosynthesis pathway } \\ \text { HMGR } & \text { 3-Hydroxy-3-methyl-glutaryl coenzyme A } \\ & \text { reductase } \\ \text { L6-GLUT4myc } & \text { Rat L6 skeletal muscle cells that stably } \\ & \text { express GLUT4 and carry an exofacial } \\ & \text { myc-epitope } \\ \text { PIP } & \text { Phosphatidylinositol 4,5-bisphosphate } \\ \text { PM } & \text { Plasma membrane } \\ \text { Sp1 } & \text { Specificity protein 1 } \\ \text { SREBP } & \text { Sterol regulatory element-binding protein } \\ \beta C D & \text { Methyl- } \beta \text {-cyclodextrin }\end{array}$

\section{Introduction}

Increased circulation of fatty acids (FAs) and accumulation of lipids in muscle contributes to glucose intolerance $[1,2]$. Several FA-induced mechanisms of this metabolic defect have been described [3]. Although it is possible that one of these dominates, a consensus is that these mechanisms are interdependent [3]. Similar to the negative consequences of FAs, hyperinsulinaemia causes metabolic derangement [4, 5], possibly because of impaired insulin-responsive GLUT4 regulation. In fact, hyperinsulinaemia-induced GLUT4 dysregulation has been documented in 3T3-L1 adipocytes and L6 myotubes $[6,7]$. Although the details remain to be defined, data from these cells suggest cortical filamentous actin (F-actin) loss, not defects in insulin signalling, as a basis of hyperinsulinaemia-induced GLUT4 dysregulation $[6,7]$. A comparable loss of F-actin is also observed in skeletal muscle obtained from insulin-resistant obese Zucker rats [7]. These findings complement an emerging appreciation in the field that, while defects in proximal insulin signalling may occur in various insulin-resistant models, distal GLUT4 defects may represent another critical node of impaired glucose metabolism [8-10].

It is well documented that insulin elicits a rapid dynamic remodelling of actin filaments into a cortical mesh, and that this mesh is necessary for GLUT4 translocation [9]. Also relevant are data that place GLUT4 storage vesicles (GSVs) in the F-actin meshwork, suggesting that this cytoskeletal structure tethers GSVs in the region beneath the plasma membrane (PM), where the final steps of GSV/PM docking and fusion are critically regulated [9]. Interestingly, cholesterol-enriched regions of the PM have been implicated in regulating F-actin structure [11-13]. While underexplored, cholesterol regulation at the PM is likely to play a very relevant role in GLUT4 regulation. For example, extraction of small $(\leq 30 \%)$ amounts of PM cholesterol enhances insulin-stimulated GLUT4 translocation [14, 15]. The cholesterol-dependent gain in PM GLUT4 resulting from a small decrease in PM cholesterol is not associated with an inhibition of endocytosis [14, 15], but this does occur with extraction of larger amounts [14-17].

These findings prompted this study to examine membrane/cytoskeletal features under in vivo and in vitro conditions of FA-induced insulin resistance. Herein, data suggest that excess FAs induce the accrual of skeletal muscle membrane cholesterol and that this provokes a loss in F-actin that plays a critical role in GLUT4 translocation. Data further indicate that the accumulation of membrane cholesterol results from increased hexosamine biosynthesis pathway (HBP) activity engaging a cholesterologenic transcriptional response. Adding to the notion that defects other than those in proximal insulin signalling contribute to insulin resistance, it was found that cholesterol lowering rescues F-actin and GLUT4 responsiveness, but not defects in insulin signalling. Together, these data highlight the hitherto unappreciated importance of membrane/cytoskeletal derangement in GLUT4 dysfunction in insulin resistance.

\section{Methods}

Animals Male C57/BL6J mice (Jackson Laboratory, Bar Harbor, ME, USA), 4 weeks old, were assigned to two groups for 4 weeks of treatment. Control mice $(n=3)$ received a diet containing 18\% energy from protein, $78 \%$ energy from carbohydrates and 4\% energy from fat (Harlan, Indianapolis, IN, USA). High-fat-fed mice $(n=3)$ received a diet containing $17 \%$ energy from protein, $43 \%$ energy from carbohydrates, and $41 \%$ energy from fat (Research Diets, 
New Brunswick, NJ, USA). Hind-limb muscles were dissected out, blotted on gauze, rinsed in $\mathrm{NaCl}$, and immersed in $4 \%$ paraformaldehyde/phosphate buffer (vol./vol.) (soleus) or frozen in liquid nitrogen (gastrocnemius). Ossabaw miniature swine (Purdue-Indiana University School of Medicine Comparison Medicine Program, Indianapolis, IN, USA), 3 months old, were assigned to two groups for 55 weeks of treatment. Control swine $(n=9)$ received a standard lean chow diet containing 22\% energy from protein, $70 \%$ energy from carbohydrates and $8 \%$ energy from fat (TestDiet, Richmond, IN, USA). Control swine ate $6.276 \mathrm{MJ} /$ day $(1,500 \mathrm{kcal} /$ day $)$ until 6 months of age, then $10.46 \mathrm{MJ} /$ day $(2,500 \mathrm{kcal} /$ day $)$ until they were killed. Similar to the methods in previous reports from Dyson et al. and Sturek et al. [18, 19], obese swine $(n=9)$ received a high-fat feed composed of lean chow supplemented with (all wt/wt): cholesterol $2.0 \%$, hydrogenated soyabean oil $47.6 \%$ (contains $56 \%$ trans FAs), corn oil 2.5\%, and sodium cholate $0.7 \%$. This mixture yielded a composition of $10 \%$ energy from protein, 17\% energy from carbohydrates and $73 \%$ energy from fat. Pigs in the obese group ate $\sim 12.550-13.390 \mathrm{MJ} /$ day $(3,000-3,200 \mathrm{kcal} /$ day $)$ until 6 months of age, then 29.290-32.640 MJ/day (7,000$7,800 \mathrm{kcal} /$ day) until they were killed. All animals were housed in individual cages/pens with a $12 \mathrm{~h}$ light-dark cycle. Water was provided ad libitum. Fasting plasma glucose and insulin were determined from blood samples drawn from conscious pigs to calculate the HOMA value for insulin resistance [18]. All animal protocols were approved by the Indiana University School of Medicine Institutional Animal Care and Use Committee.

Cell culture Rat L6 skeletal muscle cells that stably express GLUT4 and carry an exofacial myc-epitope (L6-GLUT4myc; generously obtained from A. Klip, Hospital for Sick Children, Toronto, ON, Canada) were cultured as described by McCarthy et al. [7]. All studies used myotubes 4-6 days after initiation of differentiation. Palmitate induction of insulin resistance was performed by treating cells with palmitate conjugated to FA-free BSA (MP Biomedicals, Solon, OH, USA) as detailed by Chavez et al. [20]. Briefly, palmitate was dissolved in ethanol at a concentration of $75 \mathrm{mmol} / \mathrm{l}$. For each experiment this stock was diluted $(1: 25)$ to $3 \mathrm{mmol} / \mathrm{l}$ in $1 \% \mathrm{FBS}-\mathrm{DMEM}$ containing $2 \% \mathrm{BSA}$ (wt/vol.), sonicated, and incubated at $55^{\circ} \mathrm{C}$ for $10 \mathrm{~min}$. Dilutions of this were then prepared in $1 \% \mathrm{FBS} / \mathrm{DMEM} / 2 \%$ BSA, cooled to room temperature, filter sterilised, and used to treat myotubes for $15-16 \mathrm{~h}$. To test if inhibition of glutamine:fructose-6-phosphate amidotransferase (GFAT) with 6-diazo-5-oxo-L-norleucine (DON; Sigma, St Louis, MO, USA) protected against the palmitate-induced defects, 0 or $20 \mu \mathrm{mol} / 1 \mathrm{DON}$ was included in the overnight incubation medium. Prior to all experiments, cells were serum starved for $60 \mathrm{~min}$. All acute treatments occurred during the final 5-30 min of serum starvation, as indicated.

Human biopsies Percutaneous needle biopsies of the vastus lateralis were obtained from seven individuals (five men/ two women; BMI $27.9 \pm 1.7 \mathrm{~kg} / \mathrm{m}^{2}$, [range $22.5-34 \mathrm{~kg} / \mathrm{m}^{2}$ ]; age $37.29 \pm 4.49$ years [range $19-53$ years]) in whom insulin sensitivity was determined by hyperinsulinaemiceuglycaemic clamp [21]. All individuals gave informed consent for these studies, which were approved by the Indiana University-Purdue University Institutional Review Board.

Glucose transport Cells were incubated in glucose-free buffer $\left(125 \mathrm{mmol} / 1 \mathrm{NaCl}, 5 \mathrm{mmol} / 1 \mathrm{KCl}, 1.8 \mathrm{mmol} / \mathrm{l} \mathrm{CaCl}_{2}\right.$, $2.6 \mathrm{mmol} / 1 \mathrm{MgSO}_{4}, 25 \mathrm{mmol} / \mathrm{l} \mathrm{HEPES}, 2 \mathrm{mmol} / 1$ pyruvate, $2 \% \mathrm{BSA}$ ) for $30 \mathrm{~min}$, then either left in the basal state or stimulated with $100 \mathrm{nmol} / \mathrm{l}$ insulin for $20 \mathrm{~min}$ as described by McCarthy et al. [7]. Uptake was initiated with the addition of 2-deoxy[1,2,- $\left.{ }^{3} \mathrm{H}\right]$ glucose $(2.035 \mathrm{kBq} / \mu \mathrm{l}$; Perkin Elmer, Boston, MA, USA). Non-specific uptake was quantified via cell-associated radioactivity in the presence of $20 \mu \mathrm{mol} / \mathrm{l}$ cytochalasin B. After $5 \mathrm{~min}$, uptake was terminated via four quick washes with ice-cold PBS. Cells were solubilised in $1 \mathrm{~mol} / 1 \mathrm{NaOH}$ and $\left[{ }^{3} \mathrm{H}\right]$ was measured by liquid scintillation. Counts were normalised to total cellular protein, as determined by the Bradford method.

Membrane analyses A PM-enriched fraction was prepared as described by Khayat et al. [22]. Briefly, myotubes were harvested in a homogenisation buffer $(250 \mathrm{mmol} / 1$ sucrose, $20 \mathrm{mmol} / 1 \mathrm{HEPES}, 2 \mathrm{mmol} / 1 \mathrm{EGTA}$ and $3 \mathrm{mmol} / \mathrm{I} \mathrm{NaN}_{3}$, $\mathrm{pH}$ 7.4) containing freshly added protease inhibitors (200 $\mu \mathrm{mol} / 1 \mathrm{PMSF}, 1 \mu \mathrm{mol} / 1$ leupeptin and $1 \mu \mathrm{mol} / 1$ pepstatin A) and homogenised through a 22 gauge needle ten times. The homogenate was centrifuged at $760 \mathrm{~g}$ for 5 min at $4^{\circ} \mathrm{C}$, and the supernatant fraction was centrifuged at $31,000 \mathrm{~g}$ for $20 \mathrm{~min}$ to separate a PM-enriched pellet from an intracellular microsome supernatant fraction. The PM pellet was resuspended in homogenisation buffer. Protein and cholesterol contents were determined by the Bradford and Amplex Red methods as described by Chen et al. [14]. Several analyses revealed that changes in PM cholesterol were similarly reflected in total membrane fractions prepared by centrifuging the original homogenate at 5,000 $\mathrm{g}$ for $20 \mathrm{~min}$ and then subjecting the supernatant fraction to $100,000 \mathrm{~g}$ for $30 \mathrm{~min}$. As the number of cells required to obtain this total membrane fraction was half that required to prepare PM-enriched fractions, we used total membrane fractions.

$S D S-P A G E$ analyses Cell extracts were prepared from $10 \mathrm{~cm}$ diameter dishes. Myotubes were washed two times 
with ice-cold PBS and scraped into $1 \mathrm{ml}$ lysis buffer (25 mmol/1 Tris, pH 7.4, $50 \mathrm{mmol} / 1 \mathrm{NaF}, 10 \mathrm{mmol} / 1$ $\mathrm{Na}_{3} \mathrm{P}_{2} \mathrm{O}_{7}, 137 \mathrm{mmol} / 1 \mathrm{NaCl}, 10 \%$ glycerol, $1 \%$ Nonidet P-40) containing $2 \mathrm{mmol} / \mathrm{l} \mathrm{PMSF}, 2 \mathrm{mmol} / 1 \mathrm{Na}_{3} \mathrm{VO}_{4}, 5 \mu \mathrm{g} / \mathrm{ml}$ aprotinin, $10 \mu \mathrm{mol} / 1$ leupeptin and $1 \mu \mathrm{mol} / 1$ pepstatin $\mathrm{A}$, then rotated for $15 \mathrm{~min}$ at $4^{\circ} \mathrm{C}$. Insoluble material was separated from the soluble extract by centrifugation for $15 \mathrm{~min}$ at $4^{\circ} \mathrm{C}$. Protein concentrations were determined via the Bradford method and equivalent protein amounts were loaded onto a $7.5 \%$ acrylamide gel. The resolved fractions were transferred to nitrocellulose (Bio-Rad, Hercules, CA, USA). Phosphorylated-protein kinase B [PKB/Akt2], -Akt substrate of $160 \mathrm{kDa}$ (AS160), -insulin receptor (IR) and IRS1 proteins were detected with anti-phospho-Akt2 (Ser474) (Genscript, Piscataway, NJ, USA), anti-phosphoAkt substrate (PAS) (Cell Signaling, Danvers, MA, USA), and anti-phosphotyrosine (PY20) (BD Biosciences, Lexington, KY, USA), respectively. Equal loading was confirmed by Ponceau staining and immunoblot analysis with anti-Akt antibody (Stressgen Bioreagents, Victoria, BC, Canada) and anti-actin antibody (Cytoskeleton, Denver, CO, USA). All immunoblots were labelled with IRconjugated secondary antibody and analysed via the Odyssey imaging system (LI-COR, Lincoln, NE, USA).

Immunocytochemistry Labelling of isolated soleus muscle and myotubes was performed as described by McCarthy et al. [7]. Briefly, tissues were fixed for $2 \mathrm{~h}$ and then permeabilised. Paired tissue sections were rinsed three times in PBS and blocked in 5\% milk/PBS/ $0.05 \%$ Tween. Tissues were then incubated with anti-F-actin antibody and extensively washed with PBS prior to incubation for $60 \mathrm{~min}$ at room temperature in Rhodamine Red-X-conjugated donkey anti-mouse IgM. Tissues were again subjected to an extensive PBS wash and then a quick rinse in doubledistilled $\mathrm{H}_{2} \mathrm{O}$. Tissues were mounted in Vectashield and analysed via confocal microscopy (LSM 510 NLO; Zeiss, Thornwood, NY, USA). Myotubes were fixed and left unpermeabilised (L6-GLUT4myc) or permeabilised (F-actin, O-linked $N$-acetylglucosylation) in $0.2 \%$ Triton X-100/PBS and blocked in Odyssey blocking buffer. The samples were then incubated with anti-c-myc antibody (Santa Cruz Biotechnology, Santa Cruz, CA, USA), $\beta$-actin-specific mouse IgM anti-human F-actin antibody (Serotec, Oxford, UK), and anti-O-linked $N$-acetylglucosamine isolated from rat liver (RL2) antibody (Affinity Bioreagents, Golden, CO, USA). After overnight labelling, cells were washed and incubated with infrared-conjugated secondary antibodies. Images were collected and quantified with the Odyssey imaging system. Immunofluorescent intensity was normalised to Syto60 nucleic acid staining (Molecular Probes, Carlsbad, CA, USA). All microscope/ camera settings were identical between groups.
RNA isolation and RT-PCR Cells were lysed using a Qiagen (Qiagen, Valencia, CA, USA) QIAshredder and RNA was isolated using a Qiagen RNeasy mini kit. RNA $(500 \mathrm{ng}-1.0 \mu \mathrm{g})$ was reversed transcribed with the High Capacity cDNA Reverse Transcription Kit (Applied Biosystems, Foster City, CA, USA) to produce an RT-PCR template. Reactions were performed in a 96 well plate format using the ABI Prism 7000 Sequence Detection System. Each reaction contained the following: $12.5 \mu \mathrm{l}$ SYBR Green, $500 \mathrm{nmol} / 1$ of each primer, $5 \mu \mathrm{cDNA}$ (from the cDNA synthesis reaction), and RNase-free water up to a total volume of $25 \mu \mathrm{l}$. Data are expressed relative to Gapdh using the $\Delta \Delta \mathrm{C}_{\mathrm{t}}$ method. See Electronic supplementary material (ESM) Table 1 for primer sequences used to detect Acaca, Fasn, Gapdh, Hmgcr and Hmgcs1 genes.

Statistics All values are presented as means \pm SE. The significance of differences between means was evaluated by one-way repeated-measures ANOVA. Where differences among groups were indicated, the NeumannKeuls test was used for post hoc comparison between groups. Differences between two groups were analysed using the Student's $t$ test for independent samples. GraphPad Prism 5 software was used for all analyses. $p<0.05$ was considered significant.

\section{Results}

Muscle membrane cholesterol is elevated in glucoseintolerant animals and humans High-fat feeding of C57BL/6J mice for 4 weeks, which has been reported to induce insulin resistance [23-25], induced an increase in skeletal muscle membrane cholesterol compared with controls (Fig. 1a). This accrual of skeletal muscle membrane cholesterol was also observed in high-fat-fed Ossabaw swine (Fig. 1b). HOMA values shown in Fig. 1c confirm the insulin-resistant phenotype of this Ossabaw swine model [18, 19]. Human muscle biopsies from individuals across a range of insulin sensitivities revealed an inverse correlation between membrane cholesterol content and glucose disposal rate (Fig. 1d). From the mouse soleus muscle used to measure cholesterol (Fig. 1a), a thin slice was obtained to image F-actin. Fig. 1e shows that high-fat feeding was associated with a loss of F-actin. Using cultured L6 myotubes, the study next examined the insulin-desensitising effect of palmitate on GLUT4/glucose transport regulation and whether cholesterol accrual and/or F-actin loss were components of this effect.

Palmitate induces glucose transport system dysregulation in muscle cells Exposure of L6-GLUT4myc myotubes to 


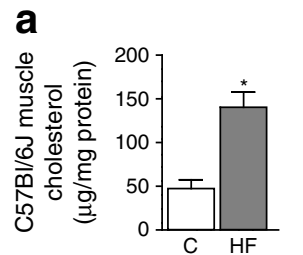

b

\section{C}
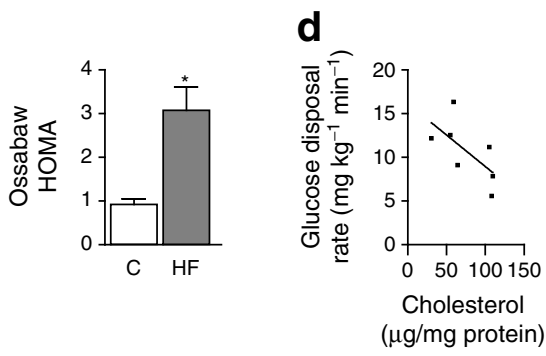

e

C57BI/6J muscle

IF: F-actin
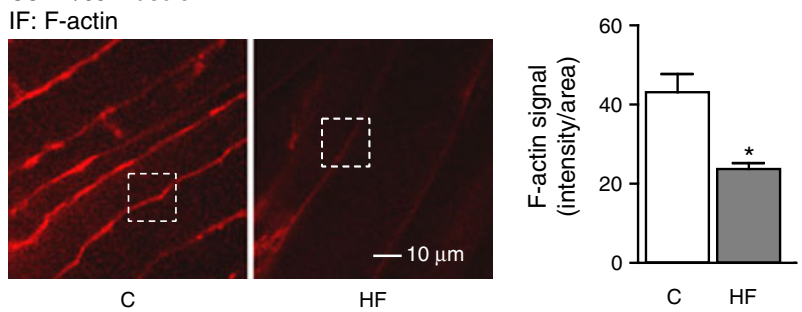

Fig. 1 Glucose-intolerant humans and animal models display skeletal muscle membrane cholesterol accrual. Skeletal muscle membrane cholesterol from control and high-fat-fed C57/B6 mice (a) and Ossabaw swine (b). HOMA values in the Ossabaw swine (c). Plot of glucose disposal rate and skeletal membrane cholesterol from humans with a range of insulin sensitivities assessed by hyperinsulinaemiceuglycaemic clamp (d); $r=-0.7857 ; p=0.048$. Cortical F-actin from skeletal muscle from control and high-fat-fed C57/B6 mice (e). Values are means \pm SE from three mice and nine swine per experimental group, and three to five images of $\mathrm{F}$-actin/area from three to five separate experiments. ${ }^{*} p<0.05$ vs control group. C, control; HF, highfat; IF, immunofluorescence. Scale bar, $10 \mu \mathrm{m}$

increasing concentrations of palmitate $(\mathrm{C} 16: 0$, a saturated FA documented to be a prevalent lipid in rat muscle [26] and to desensitise muscle and cultured myotubes to the metabolic effects of insulin $[27,28]$ ) resulted in an impairment in insulin-stimulated L6-GLUT4myc translocation (Fig. 2a). Recapitulating the findings of others, the palmitate-induced defect was significant at $300 \mu \mathrm{mol} / 1$, a concentration in the upper range of human physiology $[29$, 30]. Whereas higher concentrations of this lipid were associated with a recognised increase in basal GLUT4 translocation and glucose transport (data not shown), this was not the case with the physiological concentration of palmitate (Fig. 2b). All subsequent investigations of insulin action and membrane/cytoskeletal features used $300 \mu \mathrm{mol} / \mathrm{l}$ palmitate. Consistent with the negative effect of palmitate on insulin-regulated GLUT4 translocation, insulin-stimulated glucose transport was reduced by $36 \%$ (Fig. 2c).

Palmitate-induced insulin-resistant myotubes display increased membrane cholesterol Analogous to the animal and human findings, membranes prepared from the palmitate-induced insulin-resistant myotubes displayed a $27 \%$ increase in cholesterol and a 33\% decrease in F-actin as compared with controls (Fig. 3a, b). Removal of this excess cholesterol with a low dose $(0.1 \mathrm{mmol} / \mathrm{l})$ of methyl$\beta$-cyclodextrin $(\beta C D)$ decreased membrane cholesterol in palmitate-treated myotubes to levels $14 \%$ lower than control (Fig. 3a). As seen in Fig. 3b, this $\beta C D$-induced lowering of membrane cholesterol increased F-actin above control by $10 \%(p=0.159$ [i.e. trend]). Propidium iodine staining suggested the low dose of $\beta C D$ did not affect cell viability (ESM Fig. 1). Importantly, the cholesterol lowering/normalisation of F-actin fully restored insulinstimulated GLUT4 translocation and glucose transport in palmitate-treated myotubes (Fig. 3c, d).

Exogenously added cholesterol promotes F-actin loss and GLUT4 dysregulation Exogenous-cholesterol-loading experiments were performed to confirm directly the negative impact of excess membrane cholesterol on F-actin and glucose transport regulation. Treatment of control myotubes with $\beta C D$ conjugated to cholesterol $(\beta C D: C h o l)$
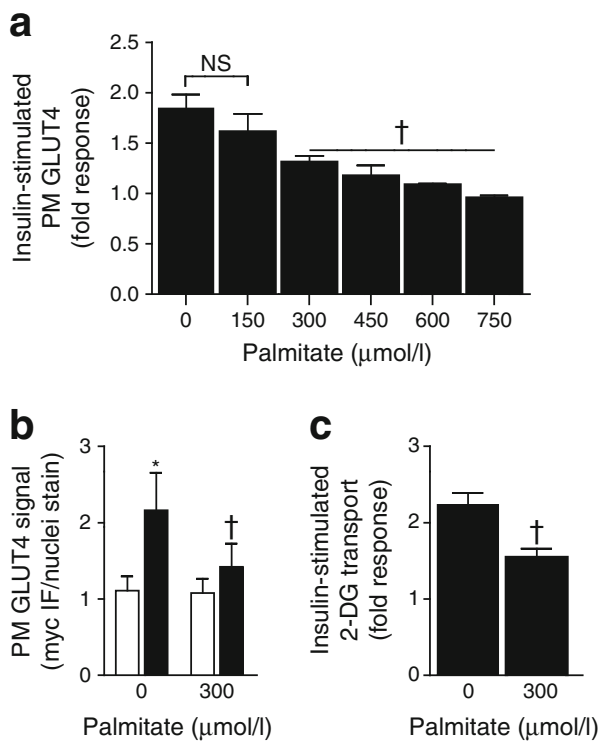

Fig. 2 Palmitate induces insulin resistance in L6 myotubes. Incubation of L6 myotubes in the presence of palmitate (C16:0) for $16 \mathrm{~h}$ impairs insulin-stimulated GLUT4 translocation (a, b) and 2-deoxyglucose uptake (c). White bars, basal; black bars, insulinstimulated for $20 \mathrm{~min}$. Values are means \pm SE from 3-12 separate experiments. ${ }^{*} p<0.05$ vs control group; ${ }^{\dagger} p<0.05$ vs control insulin group. 2-DG, 2-deoxyglucose; IF, immunofluorescence 
a

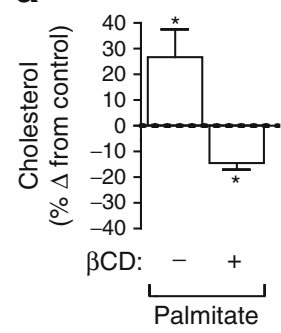

b

C

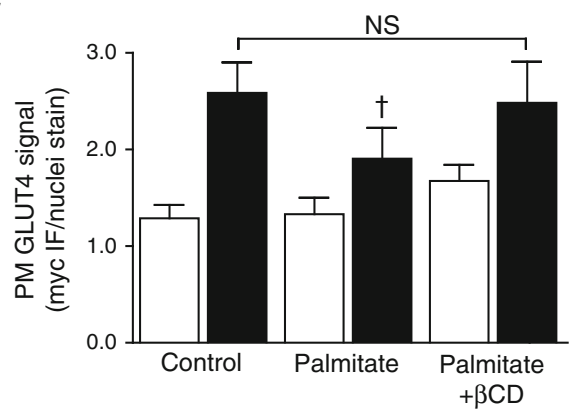

d

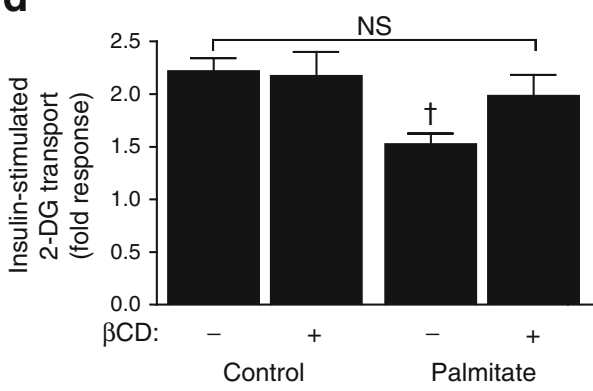

Fig. 3 Cholesterol lowering improves cortical F-actin and insulin responsiveness impaired by palmitate. Membrane cholesterol (a), cortical F-actin (b), L6-GLUT4myc (c) and 2-deoxyglucose uptake (d) from control and palmitate-treated L6 myotubes co-treated without or with $\beta C D$. White bars, basal; black bars, insulin-stimulated for $20 \mathrm{~min}$. Values are means $\pm \mathrm{SE}$ from 3-28 separate experiments. ${ }^{*} p<0.05$ vs control basal group; ${ }^{\dagger} p<0.05$ vs control insulin group. 2 -DG, 2-deoxyglucose; IF, immunofluorescence

increased membrane cholesterol (Fig. 4a) and decreased F-actin (Fig. 4b). These defects were associated with a concomitant decrease in insulin-stimulated GLUT4 translocation and glucose transport (Fig. 4c, d). Note the loss in GLUT4 responsiveness induced by $\beta \mathrm{CD}$ :Chol was similar to that induced by palmitate $(24.64 \pm 3.968[n=14]$ vs $26.76 \pm 3.932 \%[n=44] ; p=0.7746)$. In addition, although appearing more moderate, the loss in glucose transport induced by $\beta \mathrm{CD}$ :Chol was not statistically different from that induced by palmitate $(19.59 \pm 8.778[n=3]$ vs $30.90 \pm$ $3.229 \%[n=18] ; p=0.2065)$. Admittedly, testing of the effect of $\beta C D$ :Chol on transport may have been underpowered. However, Fig. $4 \mathrm{e}$ indicates that the insulinstimulated fold response was decreased by $38 \%$ with $\beta C D$ :Chol $(p=0.1338$ [i.e. trend]) and thus, collectively, these analyses suggest that exogenous cholesterol loading induces a palmitate-like insulin resistance.

Defective signal transduction appears independent of membrane cholesterol accrual Myotubes treated with $100 \mathrm{nmol} / \mathrm{l}$ insulin for $5 \mathrm{~min}$ displayed an approximately fourfold increase in Akt2 phosphorylation at serine 474 (Fig. 5). As is well documented [28], palmitate treatment caused a decrease in phosphorylation of Akt2 (Fig. 5), the isoform primarily responsible for insulin-stimulated glucose transport [31]. Strikingly, the positive effect of $\beta C D$ mediated cholesterol removal on palmitate-impaired GLUT4 translocation and glucose transport (Fig. 3c, d) was not associated with a rescue of the palmitate-induced defect in Akt2 phosphorylation (Fig. 5). Consistent with the observations of others [8], insulin-stimulated IR and IRS-1 phosphorylation remained intact in palmitatetreated myotubes, yet in support of an uncorrectable Akt2 dysfunction, phosphorylation of the Akt2 substrate AS160 was impaired by palmitate and not corrected by $\beta C D$ (ESM Fig. 2).
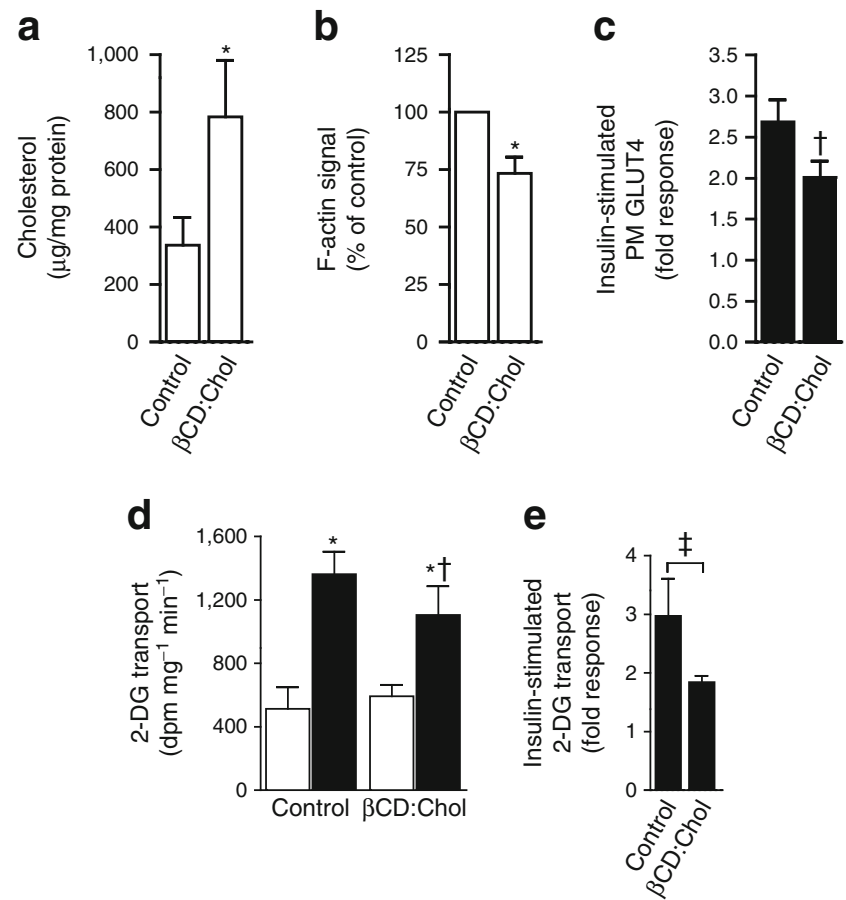

Fig. 4 Exogenous cholesterol membrane loading suppresses insulinstimulated PM GLUT4 and glucose transport. Membrane cholesterol (a), F-actin (b), L6-GLUT4myc (c) and 2-deoxylglucose uptake (d, e) in L6 myotubes treated with or without $5 \mathrm{mmol} / 1 \beta \mathrm{CD}$ :cholesterol. White bars, basal; black bars, insulin-stimulated for $20 \mathrm{~min}$. Values are means \pm SE from 3-14 separate experiments. ${ }^{*} p<0.05$ vs control basal; ${ }^{\dagger} p<0.05$ vs. control insulin group; ${ }^{\star} p=0.1338$. The significance of difference between fold insulin-stimulated 2-deoxyglucose transport values was evaluated by a paired one-tailed $t$ test. 2-DG, 2-deoxyglucose; Chol, cholesterol 
a
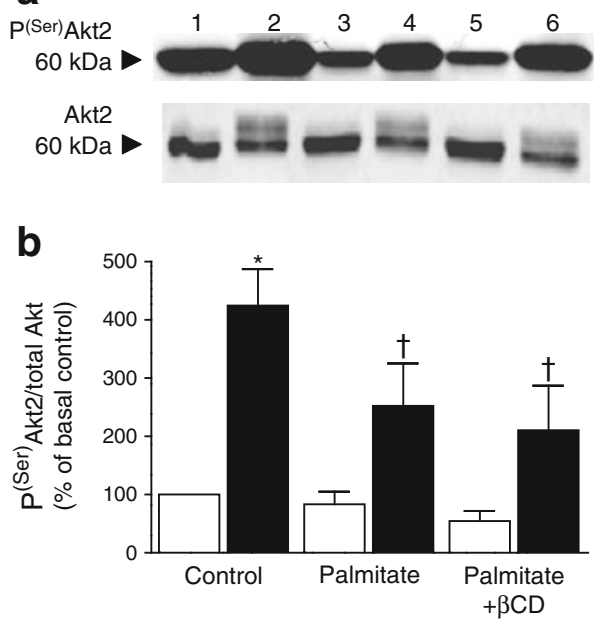

Fig. 5 Impaired insulin signalling is not rescued by cholesterol lowering. Basal and insulin-stimulated Akt2 phosphorylation in control and palmitate-treated L6 myotubes co-treated without or with $\beta \mathrm{CD}$. White bars, basal; black bars, insulin-stimulated for $5 \mathrm{~min}$. Values are means $\pm \mathrm{SE}$ from five to seven separate experiments. ${ }^{*} p<0.05$ vs control basal; ${ }^{\dagger} p<0.05$ vs control insulin group

Increased O-linked glycosylation engages a cholesterolgenic response that contributes to membrane/cytoskeletal defects and insulin resistance A previous study has shown that lipid infusion in rats [32] and direct palmitate treatment in

a
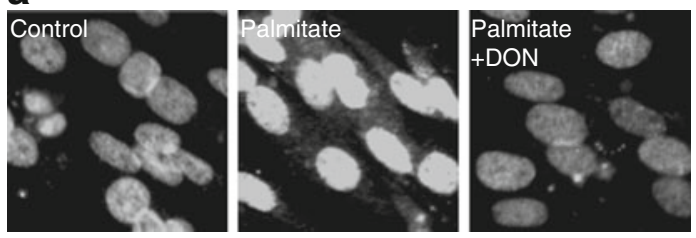

d
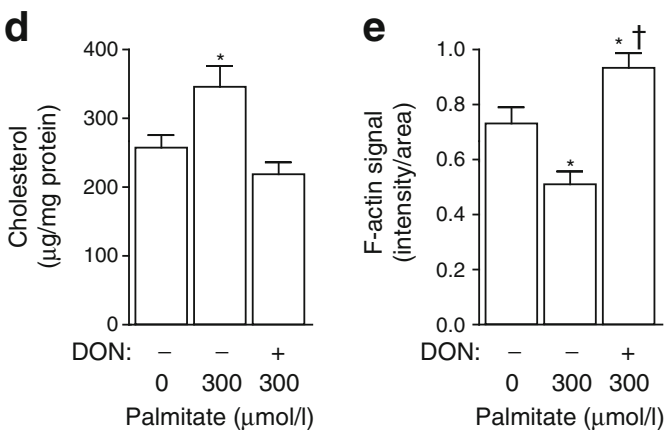

Fig. 6 Palmitate induces HBP flux and Hmgr transcription in L6 myotubes. a, b Anti-RL2-detected O-linked glycosylation immunofluorescence in: control myotubes, palmitate-treated myotubes and palmitate and DON-treated myotubes. Hmgr transcription (c), membrane cholesterol (d), cortical F-actin (e) and insulin-stimulated 2- myotubes [33] elevates glucose flux through the HBP. Moreover, the HBP has recently been implicated in hyperinsulinaemia-induced membrane cholesterol accrual, F-actin loss and insulin resistance in 3T3-L1 adipocytes [34]. Palmitate-treated myotubes displayed a $24 \%$ increase in O-linked glycosylation, an end-product of the HBP, as determined via anti-RL2 immunofluorescence (Fig. 6a, b). With regard to cholesterol synthesis, it has been shown that HBP activity increases specificity 1 (Sp1) DNA-binding activity [33]. A prediction of this modification is induction of FA synthetic and, albeit to a lesser extent, cholesterol synthetic genes [35] through Sp1-mediated coactivation of sterol regulatory element-binding protein (SREBP)-1c [36]. The transcript level of Hmgcr, which encodes 3-hydroxy-3methyl-glutaryl coenzyme A reductase (HMGR), a ratelimiting enzyme in cholesterol synthesis, was elevated by palmitate (Fig. 6c). Use of an antibody that recognises HMGR also suggested that palmitate-treated cells displayed a corresponding elevation in HMGR protein, yet the intensity of the bands detected was too low for accurate analysis. Inhibition of palmitate-induced HBP activity with the HBP inhibitor 6-diazo-5-oxo-L-norleucine (DON) lowered O-linked glycosylation, Hmgcr transcript and membrane cholesterol to or below levels observed in control cells (Fig. 6ad). This was accompanied by an increase in F-actin and a correction of insulin-regulated glucose transport (Fig. 6e, f).
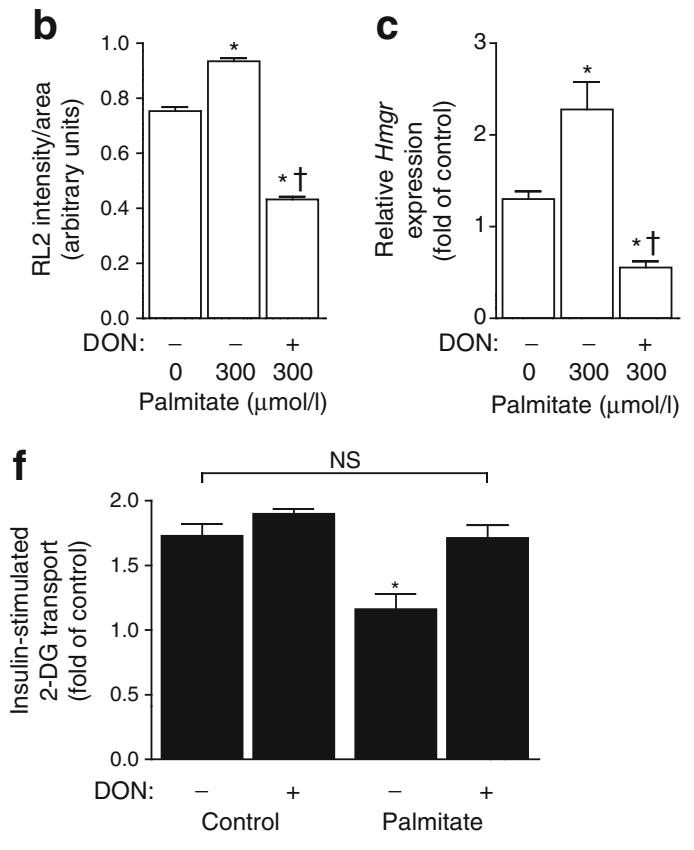

deoxyglucose transport (f) from control and palmitate-treated L6 myotubes in the absence or presence of DON. Images were taken at $\times 60$ magnification. Values are means \pm SE from 3-13 separate experiments. ${ }^{*} p<0.05$ vs non-treated control; ${ }^{\dagger} p<0.05$ vs non-treated palmitate group 


\section{Discussion}

While the derangements that contribute to glucose intolerance are certainly numerous and complex, work throughout the field has highlighted the detrimental effects of saturated FAs, and particularly palmitate, on glucose metabolism in skeletal muscle. Data presented here suggest that membrane cholesterol accrual may play a contributing role in obesityassociated glucose intolerance. Mechanistically, the cholesterol-laden membrane compromises F-actin structure, documented by several laboratories to be an essential feature of insulin and GLUT4 action [6, 7, 37-39]. Data also suggest increased HBP activity as a molecular basis for the membrane cholesterol accrual via engagement of a cholesterolgenic programme.

Interestingly, the palmitate-induced decrease in insulin signalling to Akt2 and AS160 was not as damaging as would be predicted or not yet advanced to a level to compromise GLUT4 regulation. Surprisingly, correction of the membrane/cytoskeletal defect completely restored the palmitate-induced defect in GLUT4/glucose transport regulation, but not the Akt2/AS160 impairment. This view is supported by recent analyses showing that the maximal effect of insulin on GLUT4 translocation in L6 myotubes occurs at insulin concentrations where only $5 \%$ of the total Akt pool is phosphorylated [8]. Moreover, treatments with lower palmitate concentrations $(<300 \mu \mathrm{mol} / \mathrm{l})$ were found to still impair insulin-regulated GLUT4 translocation, yet insulin signalling remained intact. Therefore, these data support a membrane/cytoskeletal defect beyond proximal insulin signalling as a major GLUT4/glucose transport system deregulator.

With regard to the cholesterolgenic model of insulin resistance suggested herein, while we found a significant elevation in the expression of $\mathrm{Hmgcr}$ with palmitate, the expression of other SREBP-1-regulated genes (e.g. Acaca, Fasn and Hmgcs l) did not consistently follow the same pattern of expression (ESM Fig. 3). For example, whereas the transcript level of Acaca was increased by palmitate, levels of Fasn and Hmgcsl were not. These variations may result from the differing contributions of the two splice variants of SREBP-1 (1a and 1c) in the control of lipogenic transcription, as it has been documented that SREBP-1a and SREBP-1c have differing levels of control over these genes [40, 41]. These experiments also found that DON was ineffective in preventing the palmitate-induced increase in Acaca. Unfortunately, we did not measure diacylglycerol or triacylglycerol levels. Nevertheless, DON treatment prevented the palmitate-induced cholesterolgenic response, lending support to a membrane cholesterol-induced, rather than a lipid-induced, state of insulin resistance. Moreover, removal of the excess membrane cholesterol with $\beta C D$ prevented the membrane/cytoskeletal defect and GLUT4/ glucose transport dysregulation. Interestingly, this tactic did not restore insulin signalling, perhaps suggesting that an increase in intramyocellular lipids is occurring and is the basis for the palmitate-induced impairment in insulin signalling. These observations suggest high-fat-feedinginduced cholesterol, but not diacylglycerol/triacylglycerol, accrual compromises membrane/cytoskeletal mechanisms important for glucose transport.

The concept that increased HBP activity might provoke insulin resistance via a SREBP response is supported by recent data showing that inhibition of SREBP improves insulin resistance in high-fat-fed mice [42]. Whether this is a SREBP/HMGR response is not clear, but our work would suggest this to be a possibility. In addition to HBP activity possibly increasing SREBP transcript via O-linked $N$-acetylglucosylation of $\mathrm{Sp} 1[33,36]$, the HBP has also been implicated in increased liver $\mathrm{X}$ receptor-dependent activation of the SREBP-1c promoter [43]. Given the complexity of the transcriptional control of cholesterol synthesis, nuclear analyses dissecting how the HBP provokes membrane cholesterol toxicity are currently under way.

It should be noted that the high-fat diets we employed for the swine and mice contained $2 \%$ and $0.2 \%$ cholesterol, respectively. This difference in dietary cholesterol amount did not equate to a similar difference in muscle membrane cholesterol accrual. For example, the high-fat-fed swine that consumed more cholesterol displayed a lower increase in muscle membrane cholesterol than the mice fed a highfat diet containing far less cholesterol. As mammalian cells contain an intricate feedback system that senses the level of membrane cholesterol and modulates the transcription of genes that mediate cholesterol synthesis and uptake [44], it is likely that circulating cholesterol does not contribute to muscle membrane cholesterol content. In fact, these data seem to collectively place the HBP as a central participant in peripheral cholesterol toxicity.

It is possible that the F-actin changes are localised in cholesterol-enriched caveolae microdomain membrane regions. Intriguingly, imaging analyses from this study support the observed reciprocal changes in membrane cholesterol and F-actin. Notably, F-actin labelling has been documented in electron micrographs to be localised in caveolae regions [45]. While caveolae have been postulated to contribute to many functions in insulin and GLUT4 action through the years [9], these findings must be cautiously interpreted. Concerns regarding the study of caveolae are associated with each of the numerous strategic approaches used to study these structures. In spite of these caveats, fluorescence confocal labelling of caveolae and F-actin have revealed actin filaments emanating from caveolae microdomains [11]. Moreover, quantitative electron microscopy and freeze-fracture analyses have revealed that cytoskeletal components, including actin, are highly 
enriched in the membrane area underlying the neck part of caveolae [12]. These findings assign caveolae a critical role in the functionality of F-actin organisation. Given the unequivocal importance of $\mathrm{F}$-actin in insulin-regulated GLUT4 translocation, these findings also emphasise the importance of caveolae in GLUT4 regulation.

Of interest to our understanding of caveolaeassociated actin regulation are new electron microscopic data showing high concentrations of phosphatidylinositol 4,5-bisphosphate $\left(\mathrm{PIP}_{2}\right)$ at the rim of caveolae [46]. This localisation of $\mathrm{PIP}_{2}$ is consistent with its regulation of the cytoskeleton where the availability of this lipid is recognised to modulate membrane/cytoskeleton interaction, the stability of F-actin and the turnover of stress fibres [47]. Interestingly, reduced $\mathrm{PM} \mathrm{PIP}_{2}$ and F-actin structure are observed in hyperinsulinaemia-induced insulin-resistant 3T3-L1 adipocytes and L6 myotubes. In these cells insulin-stimulated GLUT4 translocation is impaired, but can be corrected with exogenous $\mathrm{PIP}_{2}$ addition to the PM that mediates a restoration of F-actin structure [6,7].

In striking similarity to our myotube findings, an approximate $40 \%$ reduction in insulin-stimulated muscle glucose transport has been seen as early as 5 weeks in C57Bl/6J mice fed a high-fat diet [23] and, at the 4 week interval, muscle insulin resistance in these animals is also suggested by a marked decrease in glucose disposal rate with no change in hepatic glucose production [24]. Interestingly, insulin-stimulated Akt phosphorylation shows a trend to be decreased by 4 weeks of high-fat feeding, though this effect did not reach statistical significance until after 8 weeks; a similar pattern was observed in liver tissue [25]. Ongoing studies are now specifically evaluating the temporal sequence of membrane/cytoskeletal and signal transduction derangements in skeletal muscle from high-fat fed animals. A prediction we favour is that membrane/ cytoskeletal derangement occurs before signal dysfunction and this early event may contribute to the initial loss of insulin sensitivity.

In summary, these data suggest that a contributing factor in the pathogenesis of glucose intolerance might involve an accrual of skeletal muscle membrane cholesterol and a resultant defect in membrane/cytoskeletal function. Interestingly, this membrane/cytoskeletal defect seems to occur concomitantly with impaired insulin signal propagation in the L6 myotube system. Nevertheless, the later derangement was not limiting, as correction of membrane cholesterol excess mitigated cytoskeletal dysfunction and GLUT4 responsiveness, while signalling remained impaired. Collectively, these findings implicate a reversible abnormality (i.e. elevated cholesterol synthesis/accrual) that may be an early defect that could be targeted to improve glucose disposal.
Acknowledgements The expertise and assistance of A. Baron (University of California, San Diego, CA, USA) are gratefully acknowledged. This work was supported by NIH grants: AT001846 (J. S. Elmendorf); DK082773 (J. S. Elmendorf); DK082773-S1 (J. S. Elmendorf); HL062552 (M. Sturek); and RR013223 (M. Sturek). In addition, this work was funded by the American Diabetes Association (M. Sturek); the Purdue-Indiana University Comparative Medicine Program (M. Sturek); the Fortune-Fry Ultrasound Research Fund of the Department of Cellular \& Integrative Physiology at Indiana University School of Medicine; Indiana University Diabetes and Obesity Research Training Program DeVault Fellowship (K. M. Habegger, E. K. Blue); and Indiana Center for Vascular Biology HL079995 (W. Sealls).

Contribution statement $\mathrm{KMH}$ was responsible for the conception and design of the study, the analyses and interpretation of data, and drafting the article and revising it critically; BAP, WS and LT were responsible for design of the study, the analyses and interpretation of data, and contributed to the final manuscript version; LNB, EKB and MAA were responsible for the analyses and interpretation of data, and contributed to the final manuscript version; PJG, MS, HOS and RVC were responsible for interpretation of data and contributed to the final manuscript version, and JSE was responsible for the conception and design of the study, interpretation of data, and drafting the article and revising it critically. All authors approved the final version of the manuscript to be published.

Duality of interest The authors declare that there is no duality of interest associated with this manuscript.

Open Access This article is distributed under the terms of the Creative Commons Attribution Noncommercial License which permits any noncommercial use, distribution, and reproduction in any medium, provided the original author(s) and source are credited.

\section{References}

1. Manco M, Mingrone G, Greco AV et al (2000) Insulin resistance directly correlates with increased saturated fatty acids in skeletal muscle triglycerides. Metabolism 49:220-224

2. Perseghin G, Scifo P, De Cobelli F et al (1999) Intramyocellular triglyceride content is a determinant of in vivo insulin resistance in humans: a ${ }^{1} \mathrm{H}_{-}{ }^{13} \mathrm{C}$ nuclear magnetic resonance spectroscopy assessment in offspring of type 2 diabetic parents. Diabetes 48:1600-1606

3. Qatanani M, Lazar MA (2007) Mechanisms of obesity-associated insulin resistance: many choices on the menu. Genes Dev 21:1443-1455

4. Rizza RA, Mandarino LJ, Genest J, Baker BA, Gerich JE (1985) Production of insulin resistance by hyperinsulinaemia in man. Diabetologia 28:70-75

5. McGarry JD (1998) Glucose-fatty acid interactions in health and disease. Am J Clin Nutr 67:500S-504S

6. Chen G, Raman P, Bhonagiri P, Strawbridge AB, Pattar GR, Elmendorf JS (2004) Protective effect of phosphatidylinositol 4,5bisphosphate against cortical filamentous actin loss and insulin resistance induced by sustained exposure of 3T3-L1 adipocytes to insulin. J Biol Chem 279:39705-39709

7. McCarthy AM, Spisak KO, Brozinick JT, Elmendorf JS (2006) Loss of cortical actin filaments in insulin-resistant skeletal muscle cells impairs GLUT4 vesicle trafficking and glucose transport. Am J Physiol Cell Physiol 291:C860-C868 
8. Hoehn KL, Hohnen-Behrens C, Cederberg A et al (2008) IRS1independent defects define major nodes of insulin resistance. Cell Metab 7:421-433

9. Hoffman NJ, Elmendorf JS (2011) Signaling, cytoskeletal and membrane mechanisms regulating GLUT4 exocytosis. Trends Endocrinol Metab 22:110-116

10. Xiong W, Jordens I, Gonzalez E, McGraw TE (2010) GLUT4 is sorted to vesicles whose accumulation beneath and insertion into the plasma membrane are differentially regulated by insulin and selectively affected by insulin resistance. Mol Biol Cell 21:1375-1386

11. Kanzaki M, Pessin JE (2002) Caveolin-associated filamentous actin (Cav-actin) defines a novel F-actin structure in adipocytes. J Biol Chem 277:25867-25869

12. Foti M, Porcheron G, Fournier M, Maeder C, Carpentier JL (2007) The neck of caveolae is a distinct plasma membrane subdomain that concentrates insulin receptors in 3T3-L1 adipocytes. Proc Natl Acad Sci USA 104:1242-1247

13. Lebreton S, Paladino S, Zurzolo C (2008) Selective roles for cholesterol and actin in compartmentalization of different proteins in the Golgi and plasma membrane of polarized cells. J Biol Chem 283:29545-29553

14. Chen G, Liu P, Pattar GR et al (2006) Chromium activates glucose transporter 4 trafficking and enhances insulin-stimulated glucose transport in 3T3-L1 adipocytes via a cholesterol-dependent mechanism. Mol Endocrinol 20:857-870

15. Liu P, Leffler BJ, Weeks LK et al (2004) Sphingomyelinase activates GLUT4 translocation via a cholesterol-dependent mechanism. Am J Physiol Cell Physiol 286:C317-C329

16. Shigematsu S, Watson RT, Khan AH, Pessin JE (2003) The adipocyte plasma membrane caveolin functional/structural organization is necessary for the efficient endocytosis of GLUT4. J Biol Chem 278:10683-10690

17. Blot V, McGraw TE (2006) GLUT4 is internalized by a cholesterol-dependent nystatin-sensitive mechanism inhibited by insulin. EMBO J 25:5648-5658

18. Dyson MC, Alloosh M, Vuchetich JP, Mokelke EA, Sturek M (2006) Components of metabolic syndrome and coronary artery disease in female Ossabaw swine fed excess atherogenic diet. Comp Med 56:35-45

19. Sturek M, Wenzel J, Byrd JP et al (2007) Ossabaw island miniture swine: cardiometabolic syndrome assessment. In: Swindle M (ed) Swine in the laboratory: surgery, anesthesia, imaging and experimental techniques. CRC, Boca Raton, pp 397-402

20. Chavez JA, Holland WL, Bar J, Sandhoff K, Summers SA (2005) Acid ceramidase overexpression prevents the inhibitory effects of saturated fatty acids on insulin signaling. J Biol Chem 280:2014820153

21. Garvey WT, Maianu L, Zhu JH, Brechtel-Hook G, Wallace P, Baron AD (1998) Evidence for defects in the trafficking and translocation of GLUT4 glucose transporters in skeletal muscle as a cause of human insulin resistance. J Clin Invest 101:2377-2386

22. Khayat ZA, Tsakiridis T, Ueyama A, Somwar R, Ebina Y, Klip A (1998) Rapid stimulation of glucose transport by mitochondrial uncoupling depends in part on cytosolic $\mathrm{Ca}^{2+}$ and cPKC. Am J Physiol 275:C1487-C1497

23. Turner N, Bruce CR, Beale SM et al (2007) Excess lipid availability increases mitochondrial fatty acid oxidative capacity in muscle: evidence against a role for reduced fatty acid oxidation in lipid-induced insulin resistance in rodents. Diabetes 56:20852092

24. de Leeuw van Weenen JE, Hu L, Jansen-Van Zelm K et al (2009) Four weeks high fat feeding induces insulin resistance without affecting dopamine release or gene expression patterns in the hypothalamus of C57B16 mice. Brain Res 1250:141-148

25. Kim F, Pham M, Maloney E et al (2008) Vascular inflammation, insulin resistance, and reduced nitric oxide production precede the onset of peripheral insulin resistance. Arterioscler Thromb Vasc Biol 28:1982-1988

26. Gorski J, Nawrocki A, Murthy M (1998) Characterization of free and glyceride-esterified long chain fatty acids in different skeletal muscle types of the rat. Mol Cell Biochem 178:113-118

27. Aas V, Rokling-Andersen M, Wensaas AJ, Thoresen GH, Kase ET, Rustan AC (2005) Lipid metabolism in human skeletal muscle cells: effects of palmitate and chronic hyperglycaemia. Acta Physiol Scand 183:31-41

28. Chavez JA, Summers SA (2003) Characterizing the effects of saturated fatty acids on insulin signaling and ceramide and diacylglycerol accumulation in 3T3-L1 adipocytes and $\mathrm{C} 2 \mathrm{C} 12$ myotubes. Arch Biochem Biophys 419:101-109

29. Vaccaro O, Mancini FP, Ruffa G et al (2002) Fasting plasma free fatty acid concentrations and Pro12Ala polymorphism of the peroxisome proliferator-activated receptor (PPAR) gamma2 gene in healthy individuals. Clin Endocrinol (Oxf) 57:481-486

30. Kim F, Tysseling KA, Rice J et al (2005) Free fatty acid impairment of nitric oxide production in endothelial cells is mediated by IKKbeta. Arterioscler Thromb Vasc Biol 25:989-994

31. Cho H, Thorvaldsen JL, Chu Q, Feng F, Birnbaum MJ (2001) Akt1/PKBalpha is required for normal growth but dispensable for maintenance of glucose homeostasis in mice. J Biol Chem 276:38349-38352

32. Hawkins M, Angelov I, Liu R, Barzilai N, Rossetti L (1997) The tissue concentration of UDP- $N$-acetylglucosamine modulates the stimulatory effect of insulin on skeletal muscle glucose uptake. J Biol Chem 272:4889-4895

33. Weigert C, Klopfer K, Kausch C et al (2003) Palmitate-induced activation of the hexosamine pathway in human myotubes: increased expression of glutamine:fructose-6-phosphate aminotransferase. Diabetes 52:650-656

34. Bhonagiri P, Pattar GR, Habegger KM, McCarthy AM, Tackett L, Elmendorf JS (2011) Evidence coupling increased hexosamine biosynthesis pathway activity to membrane cholesterol toxicity and cortical filamentous actin derangement contributing to cellular insulin resistance. Endocrinology 152:3373-3384

35. Brown MS, Goldstein JL (1997) The SREBP pathway: regulation of cholesterol metabolism by proteolysis of a membrane-bound transcription factor. Cell 89:331-340

36. Lagor WR, Heller R, de Groh ED, Ness GC (2007) Functional analysis of the hepatic HMG-CoA reductase promoter by in vivo electroporation. Exp Biol Med (Maywood) 232:353-361

37. Omata W, Shibata H, Li L, Takata K, Kojima I (2000) Actin filaments play a critical role in insulin-induced exocytotic recruitment but not in endocytosis of GLUT4 in isolated rat adipocytes. Biochem J 346:321-328

38. Brozinick JT Jr, Hawkins ED, Strawbridge AB, Elmendorf JS (2004) Disruption of cortical actin in skeletal muscle demonstrates an essential role of the cytoskeleton in glucose transporter 4 translocation in insulin-sensitive tissues. J Biol Chem 279:4069940706

39. Wang Q, Bilan PJ, Tsakiridis T, Hinek A, Klip A (1998) Actin filaments participate in the relocalization of phosphatidylinositol3kinase to glucose transporter-containing compartments and in the stimulation of glucose uptake in 3T3-L1 adipocytes. Biochem $\mathrm{J}$ 331(Pt 3):917-928

40. Horton JD, Shimomura I, Ikemoto S, Bashmakov Y, Hammer RE (2003) Overexpression of sterol regulatory element-binding protein-1a in mouse adipose tissue produces adipocyte hypertrophy, increased fatty acid secretion, and fatty liver. J Biol Chem 278:36652-36660

41. Shimomura I, Hammer RE, Richardson JA et al (1998) Insulin resistance and diabetes mellitus in transgenic mice expressing nuclear SREBP-1c in adipose tissue: model for congenital generalized lipodystrophy. Genes Dev 12:3182-3194 
42. Tang JJ, Li JG, Qi W et al (2011) Inhibition of SREBP by a small molecule, betulin, improves hyperlipidemia and insulin resistance and reduces atherosclerotic plaques. Cell Metab 13:44-56

43. Anthonisen EH, Berven L, Holm S, Nygard M, Nebb HI, Gronning-Wang LM (2010) Nuclear receptor liver X receptor is $O$-GlcNAc-modified in response to glucose. $\mathrm{J}$ Biol Chem 285:1607-1615

44. Radhakrishnan A, Goldstein JL, McDonald JG, Brown MS (2008) Switch-like control of SREBP-2 transport triggered by small changes in ER cholesterol: a delicate balance. Cell Metab 8:512-521
45. Parton RG, Molero JC, Floetenmeyer M, Green KM, James DE (2002) Characterization of a distinct plasma membrane macrodomain in differentiated adipocytes. J Biol Chem 277:46769-46778

46. Fujita A, Cheng J, Fujimoto T (2010) Quantitative electron microscopy for the nanoscale analysis of membrane lipid distribution. Nat Protoc 5:661-669

47. Kwik J, Boyle S, Fooksman D, Margolis L, Sheetz MP, Edidin M (2003) Membrane cholesterol, lateral mobility, and the phosphatidylinositol 4,5-bisphosphate-dependent organization of cell actin. Proc Natl Acad Sci U S A 100:13964-13969 (Epub 2003 Nov 11) 